\title{
Patient education, disease activity and physical function: can we be more targeted? A cross sectional study among people with rheumatoid arthritis, psoriatic arthritis and hand osteoarthritis
}

\author{
Răzvan G Drăgoi ${ }^{1,2}$, Mwidimi Ndosi $^{3}$, Martina Sadlonova', Jackie Hill ${ }^{3}$, Mona Duer ${ }^{1}$, Winfried Graninger ${ }^{4}$,
} Josef Smolen ${ }^{1}$ and Tanja A Stamm ${ }^{1,5^{*}}$

\begin{abstract}
Introduction: In order to target educational needs of patients more effectively, an Austrian-German educational needs assessment tool (OENAT) was developed, the educational needs of patients with rheumatoid arthritis (RA), psoriatic arthritis (PSA) and hand osteoarthritis ( $\mathrm{HOA}$ ) were described and the relationships between educational needs, gender, disease activity and function were explored.
\end{abstract}

Methods: The English ENAT was adapted into Austrian-German using Beaton's cross-cultural adaptation process. Internal construct validity was assessed by Rasch analysis. Educational needs across diagnostic groups and subgroups of patients were summarized descriptively and their relationship with disease activity and physical functioning explored.

Results: The sample comprised 130 RA, 125 PsA and 48 HOA patients. Their mean ages \pm SD were $56 \pm 14,51 \pm 11$ and $64 \pm 7$ years for RA, PSA and HOA; disease duration was $11 \pm 9,11 \pm 11$ and $14 \pm 9$ years, respectively. More than $70 \%$ in each patient group expressed interest in receiving education about their disease.

The educational needs differed significantly between women and men in all 3 groups. In RA and PsA, female patients expressed significantly higher educational needs than men in 'movements' and 'feelings' domains ( $p=0.04$ and $p=0.03$ for RA and $p<0.01$ and $p=0.01$ for PsA). Female patients in the HOA group had significantly higher scores on all domains except for the 'movements'. Older patients with PsA scored significantly higher than their younger counterparts in the 'pain' domain ( $p=0.05$ ). RA patients with disease duration $>5$ years), expressed higher educational needs in 'movements' ( $p<0.01)$. Educational background had effects in the PsA group only, patients with basic education had greater scores than those with higher education on 'movements' and 'arthritis process' $(p=0.01)$. In the RA group, DAS28 correlated significantly with 'movements' $(r=0.24, p=0.01)$, 'feelings' $(r=0.22, p=0.02)$, and 'treatments' $(r=0.22, p=0.03)$. In the PsA group, all OENAT domains correlated with disease activity (DAPSA and CDAl).

Conclusions: This study showed that educational needs vary with personal characteristics. Patient education may be more targeted and effective, if gender, age, educational background and disease duration are taken into account. Correlations with disease activity and function suggest that the OENAT could enable identification of 'intervention points', which can be ideal opportunities for effective patient education.

\footnotetext{
* Correspondence: tanja.stamm@meduniwien.ac.at

'Department of Rheumatology, Medical University of Vienna, Clinic of

Internal Medicine III, Vienna, Austria

${ }^{5}$ Department of Health, University of Applied Sciences FH Campus Wien,

Vienna, Austria

Full list of author information is available at the end of the article
} 


\section{Introduction}

Rheumatoid arthritis (RA) is a common systemic inflammatory disease characterized by the presence of destructive polyarthritis with a predisposition for affecting the small joints of the hand and feet [1]. RA leads to pain, swelling and stiffness, limitations in joint function as well as structural joint damage that impedes functioning in daily activities [2]. Psoriatic arthritis (PsA) is likewise an inflammatory joint disease that, aside from its association with psoriasis, manifests clinically in several ways, including arthritis, enthesitis, dactylitis, axial disease and skin/nail involvement $[3,4]$. In contrast, osteoarthritis of the hands $(\mathrm{HOA})$ is regarded a non-inflammatory joint disease and constitutes one of the most prevalent musculoskeletal diseases, leading to pain in and around affected joints, as well as bony swelling, stiffness, deformity and gradual loss of function [5]. However, HOA can also occur relatively early in life, impairing the patient's capacity to work [6]. All three above described conditions are chronic disabling diseases with impact on body functions, but also on daily activities and participation in society including productivity and employment. Although there are many definitions of patient education [7], they all indicate that it involves an interactive process between patients and professionals, aims at enabling patients' participation in treatment and improve their coping strategies. As such, patients' active participation in disease management and treatment of rheumatic diseases is essential and patient education is recommended as an integral part of the treatment [8].

The Austrian health care system does not offer any form of structured patient education to individuals with a rheumatic disease, and this role is left to the clinical groups and individual health professionals. As effective patient education needs to be patient-centered and tailored to individual educational needs [9], it is essential to assess the educational needs of patients before giving a specific type of education. Currently, there is no German tool for assessing educational needs of people with rheumatic diseases; furthermore the educational needs of people with rheumatic diseases in Austria have never been systematically explored. The Educational Needs Assessment Tool (the ENAT) exists in English and in six other European languages [10]. The aim of our study was to target educational needs of people with different rheumatic diseases more effectively. The specific aims were (i) to develop and validate an Austrian-German version of the ENAT (the OENAT), (ii) to use the OENAT to explore educational needs of people with RA, PsA and HOA and (iii) to search relationships between educational needs, gender, age, disease activity and functional ability.

\section{Methods}

\section{Design}

This study was conducted in two phases: (1) a crosscultural adaptation and validation of the OENAT, and (2) a cross-sectional survey to explore the educational needs of people with RA, PsA or HOA, their disease activity and physical functioning. The adaptation into German followed an established process for cross-cultural adaptation of self-report measures [11], which involved (i) forward translations carried out by two bilingual translators whose mother tongue was German; (ii) synthesis of the translations, carried out by the forward translators and the recording observer producing (iii) back translations, carried out by two other bilingual translators whose mother tongue was English; (iv) the expert committee meeting to review the translations, and (v) testing the pre-final OENAT on 10 patients. Following the adaptation, 303 patients with arthritis completed the OENAT and the data were subjected to Rasch analysis to assess the construct validity and reliability of the translated tool. The validated OENAT was then used in the cross-sectional survey to assess the relationship between educational needs, disease activity and function.

\section{Participants}

Patients with RA and HOA diagnosed according to the American College of Rheumatology (ACR) criteria [12,13], and patients with PsA diagnosed according to the criteria described by Moll and Wright, and by McGonagle et al. [14] were asked to participate in this study. Participation was voluntary and ethical approval was obtained from the ethical committee and internal review board of the Medical University of Vienna, Austria. Patients gave written informed consent to participate in the study and agreed that the findings of the study will be published in a scientific journal. Each patient was then asked to complete the OENAT at a routine visit to the rheumatology outpatient clinic. Patients were excluded if they (a) had any other rheumatic or neuromotor disease, (b) were unable to understand the language or study procedures or (c) were unwilling to participate.

\section{Assessments}

The ENAT is a self-report questionnaire designed to assess the educational needs of patients with rheumatic diseases [11]. It was originally developed with patients and practitioners in the UK and comprises 39 items grouped into the following seven domains: managing pain (six items), movement (five items), feelings (four items), arthritis process (seven items), treatments (seven items), self-help measures (six items) and support systems (four items) [10]. Items consist of a statement providing a Likert scale ranging from 0 (not important at all) to 4 (extremely important). The OENAT is presented in the (see Additional file 1).

In addition to the OENAT, patients completed the German version of the Health Assessment Questionnaire (HAQ) $[15,16]$, and the Clinical Disease Activity Index (CDAI) was calculated for the RA and PsA 
groups, the Simple Disease Activity Index (SDAI) for RA and the Disease Activity Index for PsA (DAPSA) [17] for the PsA group. All variables needed were recorded during the routine clinical visit at the rheumatology outpatient department.

\section{Statistical analyses}

Rasch analysis was used to assess the internal construct validity of the OENAT and its invariance to age, gender, disease duration, educational background and diagnosis. The Rasch model specifies how data should look in order to comply with fundamental requirements of measurement, for example, unidimensionality. The observed data from the ENAT were measured against the Rasch model to assess how well they fit the model. The fit-to-Rasch model implies criterion-related construct validity, reliability and statistical sufficiency [18], thus, ordinal data from a questionnaire can be converted into interval scale and analyzed using parametric statistics [19].

Differences in educational needs were explored across gender and age groups, split at the median, and patients with different disease duration and educational backgrounds. The relationships between educational needs and disease activity and physical functioning were assessed for the RA and PSA cohorts. Mean differences (MD) and Pearson correlation $\left(r_{\mathrm{p}}\right)$ with the corresponding 95\% CI were calculated, where MD of 0.2, 0.5 and 0.8; and $r$ of 0.1 , 0.3 and 0.5 represented small, moderate and large differences in correlations, respectively [20]. All analyses were performed using SPSS software version 17 [21].

\section{Results}

\section{Demographic data}

In total, 303 patients participated in our study, of whom 130 had RA, 125 PsA and 48 HOA. Their demographic data were similar to the population of patients in their respective diagnostic groups [1] (Table 1). There were significant differences in mean age between the three cohorts
$(\mathrm{F}(2)=19.08, P<0.001)$ : patients with $\mathrm{HOA}$ were older than the other two groups. There were no significant differences in disease duration $(\mathrm{F}(2)=1.10, P=0.33)$. The majority of patients (above $70 \%$ ) in each group expressed interest in receiving education about their disease (Table 1).

\section{Internal construct validity and unidimensionality of OENAT}

Rasch analysis of the OENAT revealed that the seven domains of the OENAT formed a unidimensional scale, suggesting the use of domain scores rather than individual item scores. In addition, the OENAT worked consistently across patients with different ages, gender, disease duration and educational backgrounds. The ordinal data from the OENAT were then converted into interval scale (for Rasch-transformed values see Additional file 2) and analyzed using parametric statistics [19].

\section{Educational interest and relationship to other variables}

Table 2 summarizes the educational needs of people with RA, PsA and OA by gender, age, disease duration and educational background. The significant results are indicated by asterisks in the table. Gender appears to have an effect on educational needs in all the three cohorts. In the RA group, female patients expressed higher educational needs than their male counterparts in movement $(P=0.04)$ and feelings $(P=0.03)$. Like those in the RA group, female patients with PsA scored higher on movement $(P<0.01)$ and feelings, $(P<0.01)$. Interestingly, female patients in the HOA group had significantly higher scores on all domains except on the movement domain. Age had effects on the PsA cohort only, where older patients with PsA scored higher than their younger counterparts in the pain domain, $(P=0.05)$. Disease duration had significant effects on the RA group where patients with longer disease duration ( $>5$ years), expressed higher educational needs in movements, $(P<0.01)$. Educational background had effects in the PsA but not the other cohorts. Patients with basic

Table 1 Demographic data of the three diagnostic cohorts

\begin{tabular}{|c|c|c|c|c|}
\hline & & RA & PsA & $\mathrm{HOA}$ \\
\hline \multicolumn{2}{|l|}{ Female, n (\%) } & $98(75 \%)$ & $56(45 \%)$ & $40(83 \%)$ \\
\hline \multicolumn{2}{|l|}{ Age, years, mean (SD) } & $56(13.6)$ & $51(10.5)$ & $64(7)$ \\
\hline \multicolumn{2}{|c|}{ Disease duration, years, mean (SD) } & $11(9)$ & $11(10.9)$ & $14(9.1)$ \\
\hline \multirow[t]{3}{*}{ Educational background } & Basic, n (\%) & $46(35 \%)$ & $32(26 \%)$ & $21(44 \%)$ \\
\hline & Secondary, n (\%) & $50(39 \%)$ & $66(53 \%)$ & $17(35 \%)$ \\
\hline & Above, n (\%) & $31(23.8 \%)$ & $23(18.4 \%)$ & $9(18.8 \%)$ \\
\hline \multicolumn{2}{|c|}{ Interest in receiving education } & $91(70 \%)$ & $93(74 \%)$ & $81(89 \%)$ \\
\hline \multirow[t]{4}{*}{ How much information? } & None, n (\%) & $6(4.6 \%)$ & $3(2.4 \%)$ & $2(4.2 \%)$ \\
\hline & Some things, n (\%) & $24(18.5 \%)$ & $17(13.6 \%)$ & $5(10.4 \%)$ \\
\hline & A lot of things, $n(\%)$ & $24(18.5 \%)$ & $34(27.2 \%)$ & $5(10.4 \%)$ \\
\hline & Everything, n (\%) & 74 (57\%) & 71 (56.8\%) & $36(75 \%)$ \\
\hline
\end{tabular}


Table 2 Summary of educational needs across diagnostic groups

\begin{tabular}{|c|c|c|c|c|c|c|c|c|c|c|c|c|c|c|c|c|c|}
\hline \multirow[t]{2}{*}{ Diagnoses } & \multirow[t]{2}{*}{ Domains } & \multicolumn{4}{|c|}{ Age } & \multicolumn{4}{|c|}{ Gender } & \multicolumn{4}{|c|}{ Disease duration } & \multicolumn{4}{|c|}{ Educational background } \\
\hline & & $\begin{array}{c}\text { Older, } \\
\text { mean } \\
\text { (SD) }\end{array}$ & $\begin{array}{c}\text { Younger, } \\
\text { mean } \\
\text { (SD) }\end{array}$ & $\begin{array}{c}\text { MD } \\
(95 \% \mathrm{Cl})\end{array}$ & $\begin{array}{c}P- \\
\text { value }\end{array}$ & $\begin{array}{c}\text { Male, } \\
\text { mean } \\
\text { (SD) }\end{array}$ & $\begin{array}{c}\text { Female, } \\
\text { mean } \\
\text { (SD) }\end{array}$ & $\begin{array}{c}\text { MD } \\
(95 \% \mathrm{Cl})\end{array}$ & $\begin{array}{c}P- \\
\text { value }\end{array}$ & $\begin{array}{c}\text { Longer, } \\
\text { mean } \\
\text { (SD) }\end{array}$ & $\begin{array}{c}\text { Shorter, } \\
\text { mean } \\
\text { (SD) }\end{array}$ & $\begin{array}{c}\text { MD } \\
(95 \% \mathrm{Cl})\end{array}$ & $\begin{array}{c}P- \\
\text { value }\end{array}$ & $\begin{array}{c}\text { Basic, } \\
\text { mean } \\
\text { (SD) }\end{array}$ & $\begin{array}{c}\text { Higher, } \\
\text { mean } \\
\text { (SD) }\end{array}$ & $\begin{array}{c}\mathrm{MD} \\
(95 \% \mathrm{Cl})\end{array}$ & $\begin{array}{c}P- \\
\text { value }\end{array}$ \\
\hline \multirow[t]{8}{*}{$\begin{array}{l}\mathrm{RA} \\
(\mathrm{n}=130)\end{array}$} & Pain & $\begin{array}{l}15.13 \\
(5.29)\end{array}$ & $\begin{array}{l}15.42 \\
(5.11)\end{array}$ & $\begin{array}{c}-0.28 \\
(-2.24,1.67)\end{array}$ & 0.772 & $\begin{array}{l}14.95 \\
(4.89)\end{array}$ & $\begin{array}{l}15.4 \\
(5.24)\end{array}$ & $\begin{array}{c}-0.45 \\
(-2.75,1.83)\end{array}$ & 0.693 & $\begin{array}{l}15.31 \\
(5.10)\end{array}$ & $\begin{array}{l}14.48 \\
(5.15)\end{array}$ & $\begin{array}{c}0.83 \\
(-1.51,3.17)\end{array}$ & 0.484 & $\begin{array}{l}15.39 \\
(5.17)\end{array}$ & $\begin{array}{l}15.12 \\
(5.06)\end{array}$ & $\begin{array}{c}0.27 \\
(-1.93,2.47)\end{array}$ & 0.810 \\
\hline & Movement & $\begin{array}{l}11.50 \\
(5.05)\end{array}$ & $\begin{array}{l}11.63 \\
(5.32)\end{array}$ & $\begin{array}{c}-0.12 \\
(-2.00,1.75)\end{array}$ & 0.894 & $\begin{array}{c}9.85 \\
(5.77)^{*}\end{array}$ & $\begin{array}{c}12.12 \\
(4.89)^{*}\end{array}$ & $\begin{array}{c}-2.27 \\
(-4.45,-0.10)^{*}\end{array}$ & $0.040^{*}$ & $\begin{array}{l}12.20 \\
(4.91)^{*}\end{array}$ & $\begin{array}{c}9.02 \\
(5.38)^{*}\end{array}$ & $\begin{array}{c}3.17 \\
(0.99,5.35)^{*}\end{array}$ & $0.005^{*}$ & $\begin{array}{l}12.05 \\
(4.94)\end{array}$ & $\begin{array}{l}10.08 \\
(5.78)\end{array}$ & $\begin{array}{c}1.97 \\
(-0.18,4.12)\end{array}$ & 0.073 \\
\hline & Feelings & $\begin{array}{c}8.38 \\
(5.18)\end{array}$ & $\begin{array}{c}9.14 \\
(4.27)\end{array}$ & $\begin{array}{c}-0.75 \\
(-2.44,0.93)\end{array}$ & 0.382 & $\begin{array}{c}7.23 \\
(4.80)^{*}\end{array}$ & $\begin{array}{c}9.33 \\
(4.55)^{*}\end{array}$ & $\begin{array}{c}-.2 .10 \\
(-4.04,-0.17)^{*}\end{array}$ & $0.033^{*}$ & $\begin{array}{c}9.17 \\
(4.72)\end{array}$ & $\begin{array}{c}7.42 \\
(4.59)\end{array}$ & $\begin{array}{c}1.75 \\
(-3.02,2.35)\end{array}$ & 0.087 & $\begin{array}{c}9.00 \\
(4.60)\end{array}$ & $\begin{array}{c}8.18 \\
(4.83)\end{array}$ & $\begin{array}{c}0.82 \\
(-1.09,2.75)\end{array}$ & 0.396 \\
\hline & Arthritis & $\begin{array}{l}21.96 \\
(5.28)\end{array}$ & $\begin{array}{l}22.38 \\
(5.38)\end{array}$ & $\begin{array}{c}-0.42 \\
(-2.36,1.51)\end{array}$ & 0.677 & $\begin{array}{l}21.62 \\
(5.30)\end{array}$ & $\begin{array}{l}22.44 \\
(5.34)\end{array}$ & $\begin{array}{c}-0.82 \\
(-3.07,1.42)\end{array}$ & 0.469 & $\begin{array}{l}22.19 \\
(5.54)\end{array}$ & $\begin{array}{l}21.75 \\
(4.66)\end{array}$ & $\begin{array}{c}0.44 \\
(-1.85,2.73)\end{array}$ & 0.704 & $\begin{array}{l}22.80 \\
(4.84)\end{array}$ & $\begin{array}{l}20.82 \\
(5.79)\end{array}$ & $\begin{array}{c}1.98 \\
(-0.19,4.15)\end{array}$ & 0.074 \\
\hline & Treatments & $\begin{array}{l}18.38 \\
(6.00)\end{array}$ & $\begin{array}{l}18.26 \\
(5.89)\end{array}$ & $\begin{array}{c}0.11 \\
(-2.20,2.41)\end{array}$ & 0.923 & $\begin{array}{l}16.83 \\
(5.67)\end{array}$ & $\begin{array}{l}18.87 \\
(5.94)\end{array}$ & $\begin{array}{c}-2.03 \\
(-4.66,0.59)\end{array}$ & 0.127 & $\begin{array}{l}18.19 \\
(5.83)\end{array}$ & $\begin{array}{l}18.53 \\
(6.43)\end{array}$ & $\begin{array}{c}-0.33 \\
(-3.02,2,35)\end{array}$ & 0.807 & $\begin{array}{l}18.39 \\
(5.41)\end{array}$ & $\begin{array}{l}18.09 \\
(7.22)\end{array}$ & $\begin{array}{c}0.30 \\
(-2.28,2.89)\end{array}$ & 0.816 \\
\hline & Self-help & $\begin{array}{l}16.05 \\
(5.33)\end{array}$ & $\begin{array}{l}16.67 \\
(5.06)\end{array}$ & $\begin{array}{c}0.97 \\
(-2.54,1.30)\end{array}$ & 0.536 & $\begin{array}{l}15.57 \\
(4.80)\end{array}$ & $\begin{array}{l}16.71 \\
(5.24)\end{array}$ & $\begin{array}{c}-1.13 \\
(-3.33,1.07)\end{array}$ & 0.311 & $\begin{array}{l}16.45 \\
(5.05)\end{array}$ & $\begin{array}{l}16.11 \\
(5.53)\end{array}$ & $\begin{array}{c}0.33 \\
(-1.87,2.53)\end{array}$ & 0.765 & $\begin{array}{l}16.51 \\
(5.24)\end{array}$ & $\begin{array}{l}16.20 \\
(4.95)\end{array}$ & $\begin{array}{c}1.04 \\
(-0.57,2.67)\end{array}$ & 0.78 \\
\hline & Support & $\begin{array}{l}7.45 \\
(4.10)\end{array}$ & $\begin{array}{l}7.73 \\
(3.72)\end{array}$ & $\begin{array}{c}-0.28 \\
(-1.71,1.15)\end{array}$ & 0.700 & $\begin{array}{c}6.93 \\
(3.77)\end{array}$ & $\begin{array}{c}7.86 \\
(3.92)\end{array}$ & $\begin{array}{c}-0.93 \\
(-2.62,0.75)\end{array}$ & 0.276 & $\begin{array}{c}7.94 \\
(3.90)\end{array}$ & $\begin{array}{l}6.41 \\
(3.74)\end{array}$ & $\begin{array}{c}1.52 \\
(-0.13,3.19)\end{array}$ & 0.072 & $\begin{array}{l}7.82 \\
(3.79)\end{array}$ & $\begin{array}{c}6.78 \\
(4.06)\end{array}$ & $\begin{array}{c}1.04 \\
(-0.57,2.67)\end{array}$ & 0.20 \\
\hline & $\begin{array}{l}\text { Total } \\
\text { ENAT } \\
\text { Score }\end{array}$ & $\begin{array}{c}96.61 \\
(30.18)\end{array}$ & $\begin{array}{c}101 \\
(29.69)\end{array}$ & $\begin{array}{c}-5.20 \\
(-17.79,7.38)\end{array}$ & 0.412 & $\begin{array}{c}91.18 \\
(31.71)\end{array}$ & $\begin{array}{l}102.81 \\
(28.84)\end{array}$ & $\begin{array}{c}-11.63 \\
(-25.77,2.51)\end{array}$ & 0.106 & $\begin{array}{l}101.09 \\
(29.95)\end{array}$ & $\begin{array}{c}93.85 \\
(30.00)\end{array}$ & $\begin{array}{c}7.24 \\
(-6.98,21.47)\end{array}$ & 0.314 & $\begin{array}{l}109.92 \\
(28.04)\end{array}$ & $\begin{array}{c}96.06 \\
(34.56)\end{array}$ & $\begin{array}{c}4.86 \\
(-9.10,18.83)\end{array}$ & 0.49 \\
\hline $\begin{array}{l}\text { PsA } \\
(n=125)\end{array}$ & Pain & $\begin{array}{c}17.73 \\
(4.81)^{*}\end{array}$ & $\begin{array}{c}15.50 \\
(4.80)^{*}\end{array}$ & $\begin{array}{c}2.23 \\
(0.03,4.43)^{*}\end{array}$ & $0.050^{*}$ & $\begin{array}{l}15.20 \\
(5.25)\end{array}$ & $\begin{array}{l}16.89 \\
(4.09)\end{array}$ & $\begin{array}{c}-1.69 \\
(-3.49,0.10)\end{array}$ & 0.065 & $\begin{array}{l}16.29 \\
(4.88)\end{array}$ & $\begin{array}{l}14.87 \\
(4.64)\end{array}$ & $\begin{array}{c}1.41 \\
(-0.69,3.51)\end{array}$ & 0.186 & $\begin{array}{l}16.17 \\
(0.48)\end{array}$ & $\begin{array}{l}15.51 \\
(1.31)\end{array}$ & $\begin{array}{c}0.66 \\
(-1.71,3.04)\end{array}$ & 0.581 \\
\hline & Movement & $\begin{array}{l}10.75 \\
(4.91)\end{array}$ & $\begin{array}{l}10.39 \\
(5.37)\end{array}$ & $\begin{array}{c}0.35 \\
(-1.93,2.65)\end{array}$ & 0.760 & $\begin{array}{c}9.15 \\
(5.34)^{*}\end{array}$ & $\begin{array}{c}11.86 \\
(4.86)^{*}\end{array}$ & $\begin{array}{c}-2.70 \\
(-4.59,-0.82)^{*}\end{array}$ & $0.005^{*}$ & $\begin{array}{l}10.65 \\
(5.20)\end{array}$ & $\begin{array}{c}9.65 \\
(5.25)\end{array}$ & $\begin{array}{c}1.00 \\
(-1.17,3.18)\end{array}$ & 0.365 & $\begin{array}{c}11.03 \\
(5.00)^{*}\end{array}$ & $\begin{array}{c}7.90 \\
(5.92)^{*}\end{array}$ & $\begin{array}{c}3.12 \\
(0.69,5.56)^{*}\end{array}$ & $0.012^{*}$ \\
\hline & Feelings & $\begin{array}{c}9.46 \\
(3.82)\end{array}$ & $\begin{array}{c}9.17 \\
(4.50)\end{array}$ & $\begin{array}{c}0.28 \\
(-1.58,2.16)\end{array}$ & 0.762 & $\begin{array}{c}7.99 \\
(3.96)^{*}\end{array}$ & $\begin{array}{c}10.31 \\
(4.76)^{*}\end{array}$ & $\begin{array}{c}-2.31 \\
(-3.89,-0.73)^{*}\end{array}$ & $0.004^{*}$ & $\begin{array}{c}9.20 \\
(4.21)\end{array}$ & $\begin{array}{c}8.90 \\
(4.96)\end{array}$ & $\begin{array}{c}0.29 \\
(-1.53,2.11)\end{array}$ & 0.753 & $\begin{array}{c}9.48 \\
(4.46)\end{array}$ & $\begin{array}{c}7.74 \\
(4.03)\end{array}$ & $\begin{array}{c}1.74 \\
(-0.31,3.79)\end{array}$ & 0.096 \\
\hline & Arthritis & $\begin{array}{l}21.40 \\
(4.17)\end{array}$ & $\begin{array}{l}20.67 \\
(4.92)\end{array}$ & $\begin{array}{c}0.72 \\
(-1.38,2.83)\end{array}$ & 0.491 & $\begin{array}{l}20.22 \\
(4.85)\end{array}$ & $\begin{array}{l}21.43 \\
(4.66)\end{array}$ & $\begin{array}{c}-1.21 \\
(-2.97,0.53)\end{array}$ & 0.171 & $\begin{array}{l}21.10 \\
(4.63)\end{array}$ & $\begin{array}{l}20.15 \\
(5.04)\end{array}$ & $\begin{array}{c}0.95 \\
(-1.03,2.94)\end{array}$ & 0.343 & $\begin{array}{l}21.39 \\
(4.60)\end{array}$ & $\begin{array}{l}18.60 \\
(4.40)\end{array}$ & $\begin{array}{c}2.79 \\
(0.51,5.07)\end{array}$ & 0.017 \\
\hline & Treatments & $\begin{array}{l}19.16 \\
(4.25)\end{array}$ & $\begin{array}{l}17.40 \\
(5.81)\end{array}$ & $\begin{array}{c}1.27(0.77 \\
4.28)\end{array}$ & 0.170 & $\begin{array}{l}16.90 \\
(5.72)\end{array}$ & $\begin{array}{l}18.77 \\
(5.29)\end{array}$ & $\begin{array}{c}-1.86 \\
(-3.95,0.22)\end{array}$ & 0.079 & $\begin{array}{l}17.84 \\
(5.55)\end{array}$ & $\begin{array}{l}17.56 \\
(5.48)\end{array}$ & $\begin{array}{c}0.28 \\
(-2.07,2.65)\end{array}$ & 0.809 & $\begin{array}{l}18.19 \\
(5.43)\end{array}$ & $\begin{array}{l}16.12 \\
(5.93)\end{array}$ & $\begin{array}{c}2.06 \\
(-0.63,4.77)\end{array}$ & 0.133 \\
\hline & Self-help & $\begin{array}{l}17.17 \\
(4.54)\end{array}$ & $\begin{array}{l}16.23 \\
(4.89)\end{array}$ & $\begin{array}{c}0.94 \\
(-1.12,3.01)\end{array}$ & 0.373 & $\begin{array}{l}15.84 \\
(5.02)\end{array}$ & $\begin{array}{l}17.48 \\
(4.42)\end{array}$ & $\begin{array}{c}-1.64 \\
(-3.39,0.10)\end{array}$ & 0.065 & $\begin{array}{l}16.58 \\
(4.78)\end{array}$ & $\begin{array}{l}15.88 \\
(4.75)\end{array}$ & $\begin{array}{c}0.69 \\
(-1.29,2.68)\end{array}$ & 0.491 & $\begin{array}{l}16.66 \\
(4.77)\end{array}$ & $\begin{array}{l}16.17 \\
(5.21)\end{array}$ & $\begin{array}{c}0.48 \\
(-1.78,2.76)\end{array}$ & 0.671 \\
\hline & Support & $\begin{array}{l}8.28 \\
(3.16)\end{array}$ & $\begin{array}{l}7.26 \\
(4.28)\end{array}$ & $\begin{array}{c}1.02 \\
(-0.80,2.84)\end{array}$ & 0.275 & $\begin{array}{c}6.85 \\
(4.00)\end{array}$ & $\begin{array}{c}8.15 \\
(4.10)\end{array}$ & $\begin{array}{c}-1.29 \\
(-2.80,0.20)\end{array}$ & 0.091 & $\begin{array}{l}7.53 \\
(3.89)\end{array}$ & $\begin{array}{c}7.20 \\
(4.59)\end{array}$ & $\begin{array}{c}0.32 \\
(-1.39,2.04)\end{array}$ & 0.709 & $\begin{array}{l}7.81 \\
(4.12)\end{array}$ & $\begin{array}{l}5.97 \\
(3.82)\end{array}$ & $\begin{array}{c}1.83 \\
(-0.11,3.78)\end{array}$ & 0.065 \\
\hline & $\begin{array}{l}\text { Total } \\
\text { ENAT } \\
\text { Score }\end{array}$ & $\begin{array}{c}95.67 \\
(28.52)\end{array}$ & $\begin{array}{l}105.76 \\
(24.33)\end{array}$ & $\begin{array}{c}10.09 \\
(-4.39,24.58)\end{array}$ & 0.179 & $\begin{array}{c}91.23 \\
(28.47)\end{array}$ & $\begin{array}{l}104.98 \\
(25.37)\end{array}$ & $\begin{array}{c}-13.74 \\
(-24.89,-2.60)\end{array}$ & 0.016 & $\begin{array}{c}98.13 \\
(26.82)\end{array}$ & $\begin{array}{c}94.14 \\
(30.22)\end{array}$ & $\begin{array}{c}3.99 \\
(-9.11,17.11)\end{array}$ & 0.546 & $\begin{array}{c}99.54 \\
(26.87)\end{array}$ & $\begin{array}{c}85.88 \\
(31.58)\end{array}$ & $\begin{array}{c}13.66 \\
(-1.40,28.73)\end{array}$ & 0.075 \\
\hline & Pain & & & & 0.399 & & & & $0.001^{*}$ & & & & 0.883 & & & & 0.953 \\
\hline
\end{tabular}


Table 2 Summary of educational needs across diagnostic groups (Continued)

\begin{tabular}{|c|c|c|c|c|c|c|c|c|c|c|c|c|c|c|c|c|c|}
\hline \multirow[t]{8}{*}{$\begin{array}{l}\mathrm{HOA} \\
(\mathrm{n}=48)\end{array}$} & & $\begin{array}{l}15.09 \\
(5.29)\end{array}$ & $\begin{array}{l}16.78 \\
(3.50)\end{array}$ & $\begin{array}{c}-1.69 \\
(-5.72,2.33)\end{array}$ & & $\begin{array}{c}8.22 \\
(5.27)^{*}\end{array}$ & $\begin{array}{l}17.02 \\
(3.77)^{*}\end{array}$ & $\begin{array}{c}1.77 \\
(-12.39,-5.18)^{*}\end{array}$ & & $\begin{array}{l}15.14 \\
(5.13)\end{array}$ & $\begin{array}{l}15.63 \\
(7.26)\end{array}$ & $\begin{array}{c}-0.47 \\
(-7.01,6.06)\end{array}$ & & $\begin{array}{l}15.69 \\
(5.13)\end{array}$ & $\begin{array}{l}15.56 \\
(5.30)\end{array}$ & $\begin{array}{c}0.12 \\
(-4.24,4.49)\end{array}$ & \\
\hline & Movement & $\begin{array}{l}4.57 \\
(6.21)\end{array}$ & $\begin{array}{l}2.85 \\
(2.12)\end{array}$ & $\begin{array}{c}1.72 \\
(-2.00,5.44)\end{array}$ & 0.356 & $\begin{array}{c}1.33 \\
(0.87)\end{array}$ & $\begin{array}{c}4.65 \\
(5.75)\end{array}$ & $\begin{array}{c}-3.31 \\
(-7.47,0.83)\end{array}$ & 0.115 & $\begin{array}{c}3.74 \\
(5.25)\end{array}$ & $\begin{array}{l}1.76 \\
(0.41)\end{array}$ & $\begin{array}{c}1.97 \\
(-4.25,8.21)\end{array}$ & 0.524 & $\begin{array}{l}4.43 \\
(5.86)\end{array}$ & $\begin{array}{l}2.31 \\
(0.81)\end{array}$ & $\begin{array}{c}2.11 \\
(-2.11,6.34)\end{array}$ & 0.318 \\
\hline & Feelings & $\begin{array}{l}9.12 \\
(5.34)\end{array}$ & $\begin{array}{c}9.61 \\
(3.68)\end{array}$ & $\begin{array}{c}-0.49 \\
(-3.87,2.88)\end{array}$ & 0.769 & $\begin{array}{c}2.64 \\
(4.28)^{*}\end{array}$ & $\begin{array}{l}10.62 \\
(3.73)^{*}\end{array}$ & $\begin{array}{c}-7.98 \\
(-10.98,-4.98)^{*}\end{array}$ & $0.001^{*}$ & $\begin{array}{c}8.64 \\
(5.06)\end{array}$ & $\begin{array}{c}9.84 \\
(2.84)\end{array}$ & $\begin{array}{c}-1.19 \\
(-7.24,4.84)\end{array}$ & 0.690 & $\begin{array}{c}9.16 \\
(4.81)\end{array}$ & $\begin{array}{c}9.59 \\
(5.44)\end{array}$ & $\begin{array}{c}-0.42 \\
(-4.28,3.42)\end{array}$ & 0.824 \\
\hline & Arthritis & $\begin{array}{l}17.73 \\
(7.62)\end{array}$ & $\begin{array}{l}20.18 \\
(5.40)\end{array}$ & $\begin{array}{c}-2.45 \\
(-7.51,2.61)\end{array}$ & 0.332 & $\begin{array}{c}9.43 \\
(8.67)^{*}\end{array}$ & $\begin{array}{l}20.11 \\
(5.25)^{*}\end{array}$ & $\begin{array}{c}-10.68 \\
(-15.58,-5.77)^{*}\end{array}$ & $0.001^{*}$ & $\begin{array}{l}17.91 \\
(7.49)\end{array}$ & $\begin{array}{l}15.16 \\
(0.00)\end{array}$ & $\begin{array}{c}2.75 \\
(-6.14,11.65)\end{array}$ & 0.534 & $\begin{array}{l}18.31 \\
(6.83)\end{array}$ & $\begin{array}{l}18.64 \\
(8.41)\end{array}$ & $\begin{array}{c}-0.32 \\
(-5.97,5.31)\end{array}$ & 0.907 \\
\hline & Treatments & $\begin{array}{l}9.52 \\
(7.63)\end{array}$ & $\begin{array}{c}9.24 \\
(8.02)\end{array}$ & $\begin{array}{c}0.28 \\
(-5.45,6.02)\end{array}$ & 0.923 & $\begin{array}{c}3.51 \\
(2.68)^{*}\end{array}$ & $\begin{array}{l}10.75 \\
(7.76)^{*}\end{array}$ & $\begin{array}{c}-7.23 \\
(-13.31,-1.16)^{*}\end{array}$ & $0.021^{*}$ & $\begin{array}{c}9.21 \\
(7.80)\end{array}$ & $\begin{array}{c}6.40 \\
(0.00)\end{array}$ & $\begin{array}{c}2.81 \\
(-6.48,12.11)\end{array}$ & 0.542 & $\begin{array}{l}9.40 \\
(7.34)\end{array}$ & $\begin{array}{l}9.71 \\
(9.83)\end{array}$ & $\begin{array}{c}-0.30 \\
(-7.26,6.64)\end{array}$ & 0.929 \\
\hline & Self-help & $\begin{array}{l}18.98 \\
(4.98)\end{array}$ & $\begin{array}{l}20.38 \\
(3.20)\end{array}$ & $\begin{array}{c}-1.39 \\
(-4.54,1.74)\end{array}$ & 0.375 & $\begin{array}{l}14.10 \\
(7.11)^{*}\end{array}$ & $\begin{array}{l}20.43 \\
(3.08)^{*}\end{array}$ & $\begin{array}{c}-6.32 \\
(-9.58,-3.05)^{*}\end{array}$ & $0.001^{*}$ & $\begin{array}{l}19.38 \\
(5.00)\end{array}$ & $\begin{array}{l}17.79 \\
(0.00)\end{array}$ & $\begin{array}{c}1.59 \\
(-4.14,4.45)\end{array}$ & 0.590 & $\begin{array}{l}19.57 \\
(4.65)\end{array}$ & $\begin{array}{l}18.73 \\
(4.11)\end{array}$ & $\begin{array}{c}0.83 \\
(-2.76,4.44)\end{array}$ & 0.641 \\
\hline & Support & $\begin{array}{c}4.83 \\
(3.96)\end{array}$ & $\begin{array}{c}5.99 \\
(3.38)\end{array}$ & $\begin{array}{c}-1.15 \\
(-3.77,1.45)\end{array}$ & 0.375 & $\begin{array}{c}1.86 \\
(2.02)^{*}\end{array}$ & $\begin{array}{c}5.80 \\
(3.72)^{*}\end{array}$ & $\begin{array}{c}-3.93 \\
(-6.69,-1.18)^{*}\end{array}$ & $0.006^{*}$ & $\begin{array}{c}4.62 \\
(3.60)\end{array}$ & $\begin{array}{c}4.47 \\
(1.96)\end{array}$ & $\begin{array}{c}0.15 \\
(-4.14,4.45)\end{array}$ & 0.942 & $\begin{array}{l}4.86 \\
(3.59)\end{array}$ & $\begin{array}{c}6.06 \\
(4.57)\end{array}$ & $\begin{array}{c}-1.20 \\
(-4.05,1.64)\end{array}$ & 0.400 \\
\hline & $\begin{array}{l}\text { Total ENAT } \\
\text { Score }\end{array}$ & $\begin{array}{c}77.77 \\
(31.65)\end{array}$ & $\begin{array}{c}97.82 \\
(21.70)\end{array}$ & $\begin{array}{c}-20.04 \\
(-48.09,8.00)\end{array}$ & 0.155 & $\begin{array}{c}33.94 \\
(26.77)^{*}\end{array}$ & $\begin{array}{c}90.82 \\
(21.82)^{*}\end{array}$ & $\begin{array}{c}-56.88 \\
(-79.42,-34.33)^{*}\end{array}$ & $0.001^{*}$ & $\begin{array}{c}80.02 \\
(34.36)\end{array}$ & $\begin{array}{c}71.05 \\
(11.10)\end{array}$ & $\begin{array}{c}8.96 \\
(-32.91,50.84)\end{array}$ & 0.663 & $\begin{array}{c}82.17 \\
(29.18)\end{array}$ & $\begin{array}{c}78.97 \\
(41.83)\end{array}$ & $\begin{array}{c}3.20 \\
(-27.99,4.39)\end{array}$ & 0.835 \\
\hline
\end{tabular}

MD, mean difference (older-younger for age, male-female for gender, longer-shorter for disease duration and basic-higher for disease duration). $P$-value $\leq 0.05$ represents significance effects against the null hypothesis on 'no

difference'. *Statistically significant results. Older/younger age cutoff is based on disease-specific median age; shorter/longer disease duration cutoff is based on disease-specific $25^{\text {th }}$ percentile. RA, rheumatoid arthritis; PsA, psoriatic arthritis; HOA, hand osteoarthritis; ENAT, Educational Needs Assessment Tool. 
education only, had greater scores than those with higher educational background especially on movements $(P=0.01)$ and arthritis process $(P=0.01)$.

Cross-diagnosis comparison of overall educational needs was possible between the RA and PsA cohorts because the OENAT for two cohorts worked on a comparable scale [22]. There were no significant differences of overall educational needs between the RA and the PsA cohorts (Table 3).

Table 4 shows the correlations between the OENAT domains and disease activity composite measures. In the RA cohort, there were small but significant correlations between the Disease Activity Score (DAS28) and the following OENAT domains: movement $(r=0.24, P=0.01)$, feelings $(r=0.22, P=0.02)$, and treatments $(r=0.22, P=0.03)$. However, the OENAT did not correlate with the HAQ in the RA cohort. In the PsA cohort, all OENAT domains correlated with the disease activity indices for PsA (DAPSA and CDAI). In the same cohort, physical functioning (HAQ) significantly correlated with the following OENAT domains: movements $(r=0.38, P<0.01)$, feelings $(r=0.33, P=0.01)$, arthritis $(r=0.32, P=0.01)$ and support $(r=.28, P=0.03)$.

\section{Discussion}

This was the first study to explore the educational needs of patients in Austria using a standard instrument (the OENAT). A high percentage of patients in each disease group expressed interest in receiving education about their arthritis. This may be due to the fact that the Austrian health care system does not routinely offer a structured form of education to patients with rheumatic diseases. Currently, education may take place informally and it very much depends on the willingness of the health professional. The use of the OENAT may facilitate the identification of patients' priority needs and enable professionals to provide relevant education to patients. The OENAT was robust at summarizing the educational needs of people with RA, PsA and HOA, and discriminated well between different patient groups. Although the RA and PsA cohorts were comparable in their mean age, disease duration and their overall educational needs, the subgroups of patients in those two cohorts had differences in specific educational needs.

The number of female patient was higher in the RA and HOA cohorts, while the PsA cohort had almost equal gender representation, which is consistent with gender distribution in the population of patients with the specified diseases [1]. A slightly larger proportion of women showed more interest in education about disease management than men. Men with arthritis have been found to prioritize work commitments over health concerns [23] and may thus have expressed less interest in some educational needs. This may lead to the conclusion that patient education should be tailored to the needs of the participants, which may also include some gender-specific aspects.

When using the ENAT in clinical practice a clinician is able to specifically target the individual needs of a patient. Female patients had significantly more interest in the movement domain (for example, in devices that would help the patient do practical things, ways to make lifting easier, ways to save energy, getting enough rest and sleep, and ways to do things that lessen wear on the joints). A referral to a relevant health professional such as an occupational therapist may be necessary. Also, the feelings domain (for example, ways to deal with stress, moods or depression) seems to be particularly important for female patients and practitioners may want to proactively assess this domain during consultations with their female patients. This survey also suggests that written patient information leaflets should also focus on specific domains such as movements and dealing with stress, moods or depression.

Age, disease duration and educational background also had small effects in subgroups of patients, affecting only one or two domains. Interestingly, RA patients with longer disease duration ( $>5$ years) had more educational needs in the movements domain than those with a shorter duration. This may be the case for this particular patient group, but education on movement may still be as important for patients with early arthritis to go hand in hand with treatment strategies $[24,25]$.

Table 3 Comparison of educational needs between the RA and PsA cohorts

\begin{tabular}{|c|c|c|c|c|c|c|}
\hline \multirow{2}{*}{\multicolumn{2}{|c|}{ Domain (score range) }} & \multirow{3}{*}{$\begin{array}{c}\frac{\text { Psoriatic arthritis, }}{\text { mean (SD) }} \\
14.29(6.69)\end{array}$} & \multirow{3}{*}{$\begin{array}{c}\frac{\text { Rheumatoid arthritis, }}{\text { mean (SD) }} \\
13.40(6.99)\end{array}$} & \multicolumn{3}{|c|}{ Difference } \\
\hline & & & & \multirow{2}{*}{$\begin{array}{c}\text { MD }(\mathbf{9 5} \% \mathbf{C l}) \\
0.89(-0.79,2.58)\end{array}$} & \multirow{2}{*}{$\begin{array}{c}\text { t-test } \\
1.04\end{array}$} & \multirow{2}{*}{$\frac{\text { p-value }}{0.30}$} \\
\hline Pain & (0 to 24$)$ & & & & & \\
\hline Movement & (0 to 20$)$ & $9.79(5.67)$ & $11.07(5.62)$ & $-1.28(-2.67,0.11)$ & -1.81 & 0.07 \\
\hline Feelings & (0 to 16$)$ & $8.68(4.73)$ & $8.50(4.90)$ & $0.17(-1.01,1.36)$ & 0.29 & 0.78 \\
\hline Arthritis & (0 to 28$)$ & $19.44(6.89)$ & $20.65(7.65)$ & $-1.21(-3.01,0.59)$ & -1.32 & 0.19 \\
\hline Treatments & (0 to 28$)$ & $15.90(7.59)$ & $15.25(8.79)$ & $0.65(-1.38,2.68)$ & 0.63 & 0.53 \\
\hline Self-help & (0 to 24$)$ & $15.76(5.90)$ & $13.86(6.82)$ & $0.91(-0.67,2.49)$ & 1.14 & 0.26 \\
\hline Support & (0 to 16$)$ & $6.83(4.40)$ & $7.00(4.30)$ & $-0.17(-1.25,0.90)$ & -0.32 & 0.75 \\
\hline Total ENAT score & (0 to 164$)$ & $90.69(32.11)$ & $90.73(33.6)$ & $-0.04(-8.18,8.10)$ & -0.01 & 0.99 \\
\hline
\end{tabular}

$\mathrm{MD}$, mean difference (Psoriatic arthritis cohort minus rheumatoid arthritis cohort). $P$-value $\leq 0.05$ represents significance effects against the null hypothesis on 'no difference'. 
Table 4 Correlations between educational needs, disease activity and function for RA and PsA cohorts

\begin{tabular}{|c|c|c|c|c|c|c|c|c|c|}
\hline \multirow[t]{2}{*}{ Cohort } & & \multicolumn{2}{|c|}{ DAS28 } & \multicolumn{2}{|c|}{ HAQ } & \multicolumn{2}{|c|}{ CDAI } & \multicolumn{2}{|c|}{ SDAI } \\
\hline & & $r_{p}$ & $P$-value & $r_{\mathrm{p}}$ & $P$-value & $r_{p}$ & $P$-value & $r_{\mathrm{p}}$ & $P$-value \\
\hline \multirow[t]{9}{*}{ RA $(n=130)$} & Pain & 0.044 & 0.662 & -0.039 & 0.689 & 0.030 & 0.689 & -0.083 & 0.403 \\
\hline & Movement & $0.235^{*}$ & $0.013^{*}$ & 0.175 & 0.056 & $0.139^{*}$ & 0.130 & -0.086 & 0.368 \\
\hline & Feelings & $0.216^{*}$ & $0.023^{*}$ & $0.109^{*}$ & 0.235 & 0.054 & 0.557 & -0.005 & 0.954 \\
\hline & Arthritis & 0.086 & 0.375 & -0.044 & 0.635 & -0.025 & 0.786 & $-0.218^{*}$ & $0.023^{*}$ \\
\hline & Treatments & $0.224^{*}$ & $0.028^{*}$ & 0.014 & 0.891 & 0.082 & 0.409 & $-0.168^{*}$ & 0.099 \\
\hline & Self help & $0.169^{*}$ & 0.087 & -0.083 & 0.380 & 0.011 & 0.91 & -0.024 & 0.808 \\
\hline & Support & $0.106^{*}$ & 0.285 & 0.089 & 0.342 & -0.037 & 0.697 & -0.079 & 0.418 \\
\hline & Total ENAT score & $0.174^{*}$ & 0.115 & 0.019 & 0.860 & 0.030 & 0.777 & $-0.130^{*}$ & 0.236 \\
\hline & & \multicolumn{2}{|c|}{ DAPSA } & \multicolumn{2}{|c|}{ HAQ } & \multicolumn{2}{|c|}{ CDAl } & & \\
\hline \multirow[t]{8}{*}{ PsA $(n=125)$} & Pain & $0.255^{*}$ & $0.058^{*}$ & $0.113^{*}$ & 0.391 & $0.260^{*}$ & $0.044^{*}$ & & \\
\hline & Movement & $0.470^{*}$ & $0.000^{*}$ & $0.384^{*}$ & $0.002^{*}$ & $0.509^{*}$ & $0.000^{*}$ & & \\
\hline & Feelings & $0.317^{*}$ & $0.013^{*}$ & $0.325^{*}$ & $0.009^{*}$ & $0.303^{*}$ & $0.015^{*}$ & & \\
\hline & Arthritis & $0.406^{*}$ & $0.002^{*}$ & $0.319^{*}$ & $0.011^{*}$ & $0.422^{*}$ & $0.001^{*}$ & & \\
\hline & Treatments & $0.330^{*}$ & $0.013^{*}$ & $0.148^{*}$ & 0.259 & $0.358^{*}$ & $0.005^{*}$ & & \\
\hline & Self help & $0.361^{*}$ & $0.004^{*}$ & $0.137^{*}$ & 0.276 & $0.319^{*}$ & $0.010^{*}$ & & \\
\hline & Support & $0.377^{*}$ & $0.004^{*}$ & $0.283^{*}$ & $0.026^{*}$ & $0.376^{*}$ & $0.003^{*}$ & & \\
\hline & Total ENAT score & $0.438^{*}$ & $0.002^{*}$ & $0.282^{*}$ & $0.043^{*}$ & $0.455^{*}$ & $0.001^{*}$ & & \\
\hline
\end{tabular}

$r_{\mathrm{p}}=$ Pearson correlation, where $r=0.1,0.3$ and 0.5 represent small, moderate and large effects. $P$-value $\leq 0.05$ represents significance effects against the null hypothesis on 'no correlation'; * significant results. RA, rheumatoid arthritis; PsA, psoriatic arthritis; ENAT, Educational Needs Assessment Tool; DAS28; Disease Activity Score 28; HAQ; Health Assessment Questionnaire; CDAl; Clinical Disease Activity Index; SDAl: Simple Disease Activity Index; DAPSA, Disease Activity Index for Psoriatic Arthritis.

As expected, the educational needs in both cohorts correlated with disease activity, which indicated that patients with more active disease were more interested in education. Patients may particularly seek education in states when they are more affected by their disease. The OENAT could be used to identify time points when patients are most interested, and therefore, more receptive in receiving education; showing that this may then be an ideal opportunity for effective patient education. However, further research is needed because this study was cross-sectional and conducted at one time-point without an educational intervention. Although both the RA and PsA cohorts had comparable disease duration and mean age, it was interesting that the OENAT correlated with physical functioning in the PsA cohort only, the reason for which also needs further investigation. One reason could be that PsA can manifest itself in various different forms, such as monoarthritis, asymmetrical oligoarthritis, symmetrical or asymmetrical polyarthritis $[3,26,27]$.

The limitation of our study is that all our data were collected from convenience samples of patients attending the clinic at one center in Austria. Cross-diagnosis comparisons of educational needs were not possible for the HOA cohort due to a small number of patients, and the fact that the OENAT for OA has slightly different properties. Another limitation is that we included only the variables of gender, age, educational background and disease duration, to characterize our patients. However, other variables could also have influenced the educational needs of patients, for example, need for support, living situation, comorbidities et cetera. Further studies with larger sample sizes are required for more detailed subgroup analyses, for example, between women with oligoarthritis and men with polyarthritis et cetera.

\section{Conclusion}

The OENAT has been proved to be robust in assessing the educational needs of people with RA, PsA and HOA. It is a useful instrument to guide physicians and health professionals in the development of targeted patient-centered educational programs for patients with arthritis. The assessment of the needs of individual patients and the comparison of educational needs across subgroups of patients enables clinicians and researchers to better understand patients' needs and plan education strategies more effectively. Correlation with disease activity and function, suggest that the OENAT can enable identification of intervention points, which can be ideal opportunities for effective patient education.

Educational needs of patients with arthritis vary with personal characteristics and these should be borne in mind. They depend on factors such as gender, educational level and disease duration. By using the ENAT in other countries $[10,28]$, these findings will enable future comparisons between these regions and different parts of the world. 


\section{Additional files}

Additional file 1: The adapted Austrian-German Educational Needs Assessment Tool (OENAT) questionnaire used in the present study.

Additional file 2: The Rasch-transformed scores used for the hand osteoarthritis (HOA) cohort in the study.

\section{Abbreviations}

CDAl: Clinical disease activity index; DAPSA: Disease activity index for psoriatic arthritis; DAS 28: Disease activity score 28; ENAT: Educational needs assessment tool; HAQ: Health assessment questionnaire; HOA: Hand osteoarthritis; MD: Mean difference; OENAT: Austrian-German educational needs assessment tool; PsA: Psoriatic arthritis; RA: Rheumatoid arthritis; SDAI: Simple disease activity index.

\section{Competing interests}

The authors declare that they have no competing interests.

\section{Authors' contributions}

Conception: RGD, MN, JH, WG, JS and TAS. Data acquisition: MS, MD and TAS. Data analysis: RGD, MN, MD and TAS. Manuscript draft: RGD, MN, MD and TAS. Advice on editing of the manuscript: RGD, MN, MS, JH, MD, WG, JS, TAS. All the authors have taken an active part in the study and take responsibility for its contents, read and approved the manuscript.

\section{Acknowledgements}

The cross-cultural adaptation of the ENAT was funded by European League Against Rheumatism grant (HPR011).

The author Razvan Gabriel Drăgoi benefited from a fellowship from the POSDRU/88/1.5/S/63117 grant-Doctoral fellowships for PhD students competitive in the European Research Area.

We thank Maria Skobek and Alexa Binder for their help in translating the ENAT and contacting the patients. We thank all the patients for participation in this study.

\section{Author details}

${ }^{1}$ Department of Rheumatology, Medical University of Vienna, Clinic of Internal Medicine III, Vienna, Austria. 'Department of Rehabilitation, Physical Medicine, Balneology and Rheumatology, "Victor Babeş" University of Medicine and Pharmacy Timişoara, Timişoara, Romania. ${ }^{3}$ Academic \& Clinical Unit for Musculoskeletal Nursing, Leeds Institute of Rheumatic and Musculoskeletal Medicine, University of Leeds, Leeds, UK. ${ }^{4}$ Department of Internal Medicine, Division of Rheumatology and Immunology, Medical University of Graz, Graz, Austria. ${ }^{5}$ Department of Health, University of Applied Sciences FH Campus Wien, Vienna, Austria.

Received: 5 February 2013 Accepted: 27 September 2013

Published: 20 October 2013

\section{Reference}

1. Hakim A, Clunie GPR, Haq I: Oxford Handbook of Rheumatology. 3rd edition. Oxford, United Kingdom: Oxford University Press; 2011.

2. Smolen JS, Aletaha D, Grisar JC, Stamm TA, Sharp JT: Estimation of a numerical value for joint damage-related physical disability in rheumatoid arthritis clinical trials. Ann Rheum Dis 2010, 69:1058-1064.

3. Gladman DD, Antoni C, Mease P, Clegg DO, Nash P: Psoriatic arthritis: epidemiology, clinical features, course, and outcome. Ann Rheum Dis 2005, 64:ii14-ii17.

4. Benjamin M, McGonagle D: The enthesis organ concept and its relevance to the spondyloarthropathies. Adv Exp Med Biol 2009, 649:57-70.

5. Creamer P, Hochberg MC: Osteoarthritis. Lancet 1997, 350:503-508.

6. Kloppenburg M, Stamm T, Watt I, Kainberger F, Cawston TE, Birrell FN, Petersson IF, Saxne T, Kvien TK, Slatkowsky-Christensen B, Dougados M, Gossec L, Breedveld FC, Smolen JS: Research in hand osteoarthritis: time for reappraisal and demand for new strategies. An opinion paper. Ann Rheum Dis 2007, 66:1157-1161.

7. Lorig KR, Holman HR: Self-management education: History, definition, outcomes, and mechanisms. Ann Behav Med 2003, 26:1-7.

8. Combe B, Landewe R, Lukas C, Bolosiu HD, Breedveld F, Dougados M, Emery P, Ferraccioli G, Hazes JM, Klareskog L, Machold K, Martin-Mola E,
Nielsen H, Silman A, Smolen J, Yazici H: EULAR recommendations for the management of early arthritis: report of a task force of the European Standing Committee for International Clinical Studies Including Therapeutics (ESCISIT). Ann Rheum Dis 2007, 66:34-45.

9. Koehn $\mathrm{CL}$, Esdaile JM: Patient education and self-management of musculoskeletal diseases. Best Pract Res Clin Rheumatol 2008, 22:395-405.

10. Ndosi M, Tennant A, Bergsten U, Kukkurainen ML, Machado P, Torre-Aboki J, Vlieland TP, Zangi HA, Hill J: Cross-cultural validation of the Educational Needs Assessment Tool in RA in 7 European countries. BMC Musculoskelet Disord 2011, 12:110.

11. Hardware B, Lacey E, Shewan J: Towards the development of a tool to assess educational needs in patients with arthritis. Clin Eff Nurs 2004 8:111-117

12. Arnett FC, Edworthy SM, Bloch DA, McShane DJ, Fries JF, Cooper NS, Healey LA, Kaplan SR, Liang MH, Luthra HS: The American Rheumatism Association 1987 revised criteria for the classification of rheumatoid arthritis. Arthritis Rheum 1988, 31:315-324.

13. Altman R, Alarcon G, Appelrouth D, Bloch D, Borenstein D, Brandt K, Brown C, Cooke TD, Daniel W, Gray R: The American College of Rheumatology criteria for the classification and reporting of osteoarthritis of the hand. Arthritis Rheum 1990, 33:1601-1610.

14. Helliwell P, Taylor W: Classification and diagnostic criteria for psoriatic arthritis. Ann Rheum Dis 2005, 64:ii3-ii8.

15. Brühlmann P, Stucki G, Michel BA: Evaluation of a German Version of the Physical Dimensions of the Health Assessment Questionnaire in Patients with Rheumatoid Arthritis. J Rheumatol 1994, 21:1245-1249.

16. Fries JF, Spitz PW, Young DY: The dimensions of health outcomes: the health assessment questionnaire, disability and pain scales. J Rheumatol 1982, 9:789-793.

17. Nell-Duxneuner VP, Stamm TA, Machold KP, Pflugbeil S, Aletaha D, Smolen JS: Evaluation of the appropriateness of composite disease activity measures for assessment of psoriatic arthritis. Ann Rheum Dis 2010, 69:546-549.

18. Bond TG, Fox CM: Applying the Rasch Model. Fundamental Measurement in the Human Science. 2nd edition. London: Lawrence Erlbaum Associates, Inc; 2007.

19. Wright BD, Linacre JM: Observations Are Always Ordinal - Measurements, However, Must be Interval. Arch Phys Med Rehabil 1989, 70:857-860.

20. Cohen J: A power primer. Psychol Bull 1992, 112:155-159.

21. SPSS: SPSS Statistics 17.00 for Windows. Chicago: SPSS Inc; 2008.

22. Ndosi M, Tennant A, Hill J: Validation of the Educational Needs Assessment Tool as a generic instrument in 7 European Countries. Ann Rheum Dis. doi:10.1136/annrheumdis-2013-203461.

23. Gibbs L: Identifying work as a barrier to men's access to chronic illness (arthritis) self-management programs. International Journal of Men's Health 2007, 6:143-155.

24. Combe B: Early rheumatoid arthritis: strategies for prevention and management. Best Pract Res Clin Rheumatol 2007, 21:27-42.

25. Smolen JS, Aletaha D, Bijlsma JW, Breedveld FC, Boumpas D, Burmester G, Combe B, Cutolo M, de WM, Dougados M, Emery P, Gibofsky A, GomezReino JJ, Haraoui B, Kalden J, Keystone EC, Kvien TK, McInnes I, Martin-Mola E, Montecucco C, Schoels M, van der Heijde D: Treating rheumatoid arthritis to target: recommendations of an international task force. Ann Rheum Dis 2010, 69:631-637.

26. Helliwell $P$, Marchesoni A, Peters M, Barker M, Wright V: A re-evaluation of the osteoarticular manifestations of psoriasis. Br J Rheumatol 1991, 30:339-345.

27. Mease PJ, Behrens F, Boehncke WH, Feldman SR, FitzGerald O, Gladman DD, Helliwell P, Nash P, Olivieri I, Taylor W, Tak PP: Discussion: Assessment of Psoriatic Arthritis. Ann Rheum Dis 2005, 64:ii69-ii73.

28. Meesters JJ, Vliet Vlieland TP, Hill J, Ndosi ME: Measuring educational needs among patients with rheumatoid arthritis using the Dutch version of the Educational Needs Assessment Tool (DENAT). Clin Rheumatol 2009, 28:1073-1077

\section{doi:10.1186/ar4339}

Cite this article as: Drăgoi et al:: Patient education, disease activity and physical function: can we be more targeted? A cross sectional study among people with rheumatoid arthritis, psoriatic arthritis and hand osteoarthritis. Arthritis Research \& Therapy 2013 15:R156. 